\title{
Some Cyclic Group Actions on a Homotopy Sphere and the Parallelizability of its Orbit Spaces
}

By

Jin Ho KWAK*

\section{§1. Introduction}

In this paper, we introduce a way to define a free cyclic group action on a homotopy sphere and examine the stable parallelizability of its orbit spaces. J. Ewing et al [3] answered the stable parallelizability problem for the classical lens space, that is, the orbit space of the standard sphere under a linear cyclic group action.

Let $w_{1}, w_{2}, \ldots, w_{n+1}$ be positive rational numbers. A polynomial $f\left(z_{1}, z_{2}, \ldots, z_{n+1}\right)$ is called a weighted homogeneous polynomial of type $\left(w_{1}, w_{2}, \ldots, w_{n+1}\right)$ if it can be expressed as a linear combination of monomials

$$
z_{1}^{i_{1}} z_{2}^{i_{2}} \cdots z_{n+1}^{i_{n+1}}
$$

for which $\sum_{j=1}^{n+1} i_{j} / w_{j}=1$. This is equivalent to the requirement that

$$
f\left(e^{c / w_{1}} z_{1}, e^{c / w_{2}} z_{2}, \ldots, e^{c / w_{n+1}} z_{n+1}\right)=e^{c} f\left(z_{1}, z_{2}, \ldots, z_{n+1}\right)
$$

for every complex number $c$.

Throughout this paper, we assume that all weighted homogeneous polynomials have an isolated critical point at the origin. For example, the Brieskorn polynomial

$$
f\left(z_{1}, z_{2}, \ldots, z_{n+1}\right)=z_{1}^{a_{1}}+z_{2}^{a_{2}}+\ldots+z_{n+1}^{{ }^{a}}{ }^{a+1},
$$

all $a_{i} \geq 2$, is a weighted homogeneous polynomial of weights $w=\left(a_{1}\right.$,

Communicated by N. Shimada, December 24, 1984.

* Department of Mathematics, Kyungpook National University, Taegu 635, Korea.

This work is partially supported by KOSEF Grant 1983-84. 
$\left.a_{29}, \ldots, a_{n+1}\right)$.

Set $\sum_{w}=f^{-1}(0) \cap S^{2 n+1}$, and consider the Milnor fibering $g: S^{2 n+1}-$ $\sum_{w} \longrightarrow S^{1}$ defined by

$$
g\left(z_{1}, \ldots, z_{n+1}\right)=f\left(z_{1}, \ldots, z_{n+1}\right) /\left|f\left(z_{1}, \ldots, z_{n+1}\right)\right|,
$$

then each fiber $F_{t}=g^{-1}\left(e^{i t}\right)$ is a smooth parallelizable $2 n$-dimensional manifold with the homotopy type of a bouquet of $n$-spheres. We can obtain $S^{2 n+1}-\sum_{w}$ from $F \times[0,1]$ by identifying $F \times 0$ and $F \times 1$ by a homeomorphism $h: F \longrightarrow F$ called the characteristic map. Denote the characteristic polynomial of

$$
h_{*}: H_{n}(F ; \mathbb{C}) \longrightarrow H_{n}(F ; \mathbb{C})
$$

by

$$
\Delta(t)=\text { determinant }\left(t I_{*}-h_{*}\right),
$$

where $I$ is the identity map of $F$. This characteristic map $h_{*}$ and its characteristic polynomial $\Delta(t)$ are fundamental topological invariants. Brieskorn [2] computed $\Delta(t)$ for varieties defined by Brieskorn polynomials, and Milnor and Orlik [9] did it for weighted homogeneous polynomials.

The following theorem answers whether or not the $2 n-1$ dimensional manifold $\sum_{w}=f^{-1}(0) \cap S^{2 n+1}$ is a topological sphere.

Theorem ([8], Section. 8). For $n \geq 3$, the followings are equivalent:

i) $\sum_{w}$ is a topological sphere.

ii) $H_{n-1}\left(\sum_{w}\right)=0$.

iii) The intersection pairing $H_{n}(F ; \mathbb{Z}) \otimes H_{n}(F ; \mathbb{Z}) \longrightarrow \mathbb{Z}$ has determinant \pm 1 .

iv) $\Delta(1)= \pm 1$.

Furthermore, if $\sum_{w}$ is a topological sphere, the diffeomorphism class of $\sum_{w}$ is completely determined by the signature of the intersection pairing

$$
H_{n}(F ; \mathbb{Z}) \otimes H_{n}(F ; \mathbb{Z}) \longrightarrow \mathbb{Z}
$$

if $n$ is even. If $n$ is odd, $\sum_{w}$ is

the standard sphere if $\Delta(-1)= \pm 1(\bmod 8)$, the Kervaire sphere if $\Delta(-1)= \pm 3(\bmod 8)$.

Let $\sum_{w}=f^{-1}(0) \cap S^{2 n+1}$ be a topological sphere, where $f$ is a weighted 
homogeneous polynomial of weight $w=\left(w_{1}, w_{2}, \ldots, w_{n+1}\right)$, say $w_{i}=u_{i} / v_{i}$ in irreducible form for $i=1,2, \ldots, n+1$, and let $p$ be an odd prime number relatively prime to each $u_{i}$. To define a free cyclic group $\mathbb{Z}_{p}$-action on $\sum_{w}$, choose natural numbers $b_{i}$ such that $b_{i}=h / w_{i}=h v_{i} u_{i}^{-1}$ $(\bmod p)$ for some $h \neq 0(\bmod p)$ and $\left(b_{i}, p\right)=1$ for all $i$, where $\left(b_{i}, p\right)$ denotes the greatest common divisor of $b_{i}$ and $p$. Now, we define a map $T$ on $\sum_{w}$ by

$$
T\left(z_{1}, z_{2}, \ldots, z_{n+1}\right)=\left(\zeta^{b_{1}} z_{1}, \zeta^{b_{2}} z_{2}, \ldots, \zeta^{b_{n+1}} z_{n+1}\right),
$$

where $\zeta=e^{2 \pi i / p}$. Then

$$
f\left(T\left(z_{1}, z_{2}, \ldots, z_{n+1}\right)\right)=\zeta^{h} f\left(z_{1}, z_{2}, \ldots, z_{n+1}\right) .
$$

This is a well-defined free action on $\sum_{w}$ generating the cyclic group $\mathbb{Z}_{p}$. Denote its orbit space by $L(p ; w ; b)$. Note that we may assume that $h=1(\bmod p)$, i. e., $w_{i} b_{i}=1(\bmod p)$ for all $i$ by taking a suitable generator $T$ of $\mathbb{Z}_{p}$.

\section{§2. An Algebraic Characterization of Stable Parallelizability}

Define a $\mathbb{Z}_{p}$-action on $\sum_{w} \times \mathbb{C}$ by $T^{\prime}(z, \eta)=(T(z), \zeta \eta)$, where $\zeta$ and $T(z)$ are the same as above, so that the natural projection from $\sum_{w} \times \mathbb{C}$ to $\sum_{w}$ is equivariant, that is, it commutes with the $\mathbb{Z}_{p}$-actions. By taking quotients, one can get the canonical complex line bundle $\gamma$ over $L(p ; w ; b)$. Similarly, one can get $\gamma^{b}=r \otimes r \otimes \ldots \otimes r$, $(b$ times $)$ with a $\mathbb{Z}_{p}$-action on $\sum_{w} \times \mathbb{C}$ given by $T^{\prime}(z, \eta)=\left(T(z), \zeta^{b} \eta\right)$. It can be proved easily that

$$
r^{b} \oplus r^{b_{2}} \oplus \ldots \oplus r^{b_{n+1}}=\sum_{w} \times \mathbb{C}^{n+1} / T \times T,
$$

where

$$
(T \times T)\left(z,\left(\eta_{1}, \eta_{2}, \ldots, \eta_{n+1}\right)\right)=\left(T(z),\left(\zeta^{b_{1}} \eta_{1}, \zeta^{b_{2}} \eta_{2}, \ldots, \zeta^{b_{n+1}} \eta_{n+1}\right)\right) .
$$

To reduce the question of stable parallelizability of the orbit space $L(p ; w ; b)$ to a purely algebraic one, we first describe the tangent bundle of $L(p ; w ; b)$.

Theorem 2.1. Over $L(p ; w ; b), \tau \oplus \varepsilon \oplus \mathrm{re}(\gamma)$ is isomorphic to

$$
\operatorname{re}\left(r^{b_{1}} \oplus r^{b_{2}} \oplus \ldots \oplus r^{b_{n+1}}\right),
$$

where $\tau$ denotes the tangent bundle, $\varepsilon$ the trivial 1-dimensional real bundle 
over $L(p ; w ; b)$, and re the realification of a bundle.

Proof. Let $\tau($.) denote the tangent bundle and $\nu($.$) the normal$ bundle of the space (.) in $\boldsymbol{C}^{n+1}$, then the trivial bundle $\sum_{w} \times \boldsymbol{C}^{n+1}$ is isomorphic to

$$
\tau\left(\sum_{w}\right) \oplus \nu\left(\sum_{w}\right)=\tau\left(\sum_{w}\right) \oplus \nu\left(S^{2 n+1}\right) \oplus \nu\left(f^{-1}(0)\right)
$$

over $\sum_{w}$. But $\nu\left(S^{2 n+1}\right)$ is trivial and grad $f$ is a cross section of $\nu\left(f^{-1}(0)\right)$, so that $\nu\left(f^{-1}(0)\right)=C \cdot \operatorname{grad} f$. Define

$$
\Phi: \tau\left(\sum_{w}\right) \oplus \boldsymbol{R} \oplus \boldsymbol{C} \longrightarrow \sum_{w} \times \boldsymbol{C}^{n+1}
$$

by

$$
\Phi\left(v_{z}, r, \eta\right)=(z, v+r z+\eta \operatorname{grad} f(z)),
$$

where $v_{z}$ denotes a tangent vector at $z$ and $\boldsymbol{R}, \boldsymbol{C}$ represent the trivial bundles $\boldsymbol{R} \times \sum_{w}, \boldsymbol{C} \times \sum_{w}$ respectively. By using $\zeta \operatorname{grad} f(T z)=T(\operatorname{grad}$ $f(z))$, we can see that $\Phi$ is an equivariant isomorphism from $\tau\left(\sum_{w}\right)$ $\oplus \boldsymbol{R} \oplus \boldsymbol{C}$ with $\boldsymbol{Z}_{p}$-action given by $d T \oplus I \oplus(\cdot \zeta)$ to $\sum_{w} \times \boldsymbol{C}^{n+1}$ with $\boldsymbol{Z}_{p^{-}}$ action given by $T \times T$. Therefore, by taking quotients, it is proved.

Remark. In Theorem 2. 1, if $L(p ; w ; b)$ is defined as an orbit space of a Brieskorn sphere, then we have

$$
\tau \oplus \varepsilon \oplus \operatorname{re}(\gamma) \simeq \operatorname{re}\left(\gamma^{b} \oplus \gamma^{b_{2}} \oplus \ldots \oplus \gamma^{b+1}\right) .
$$

This is the correction of Orlik's theorem 3 ([12], p. 252).

Recall that the standard lens space $L^{2 n-1}(p)$ is defined as the orbit space of $S^{2 n-1}$ by the linear action. Since the principal $\boldsymbol{Z}_{p}$-bundles

$$
S^{2 n-1} \longrightarrow L^{2 n-1}(p) \text { and } \sum_{w} \longrightarrow L(p ; w ; b)
$$

are $2 n-1$ universal, there are maps

$$
f: L^{2 n-1}(p) \longrightarrow L(p ; w ; b) \text { and } g: L(p ; w ; b) \longrightarrow L^{2 n-1}(p)
$$

such that the induced bundles $f^{*} \gamma=\gamma$ and $g^{*} \gamma=\gamma$, where $\gamma$ is the canonical bundle over the suitable orbit space. Hence, Theorem 2.1 implies the following:

Lemma 2.2. The space $L(p ; w ; b)$ is stably parallelizable if and only if $\mathrm{re}(\gamma)$ is stably isomorphic to

$$
\mathrm{re}\left(\gamma^{b_{1}}\right) \oplus \mathrm{re}\left(\gamma^{b_{2}}\right) \oplus \ldots \oplus \operatorname{re}\left(\gamma^{b}{ }^{n+1}\right)
$$


over the standard lens space $L^{2 n-1}(p)$, where $\gamma$ represents the canonical bundle over $L^{2 n-1}(p)$.

Recall that the mod $p$ cohomology ring of the standard lens space $L^{2 n-1}(p)$ is the tensor product

$$
H^{*}\left(L^{2 n-1}(p) ; \boldsymbol{Z}_{p}\right) \simeq \Lambda(u) \otimes \boldsymbol{Z}_{p}[v] /\left(v^{n}\right)
$$

of the exterior algebra $\Lambda(u)$ and the truncated polynomial ring generated by $v$, where $\operatorname{deg} u=1, \operatorname{deg} v=2$, and $\beta^{*}{ }_{p}(u)=v$ for the Bockstein isomorphism

$$
\beta^{*}{ }_{p}: H^{1}\left(L^{2 n-1}(p) ; \mathbb{Z}_{p}\right) \longrightarrow H^{2}\left(L^{2 n-1}(p) ; \mathbb{Z}_{p}\right) .
$$

Lemma 2.3. If the space $L(p ; w ; b)$ is stably parallelizable, then

$$
1+v^{2}=\left(1+b_{1}{ }^{2} v^{2}\right)\left(1+b_{2}{ }^{2} v^{2}\right) \cdots\left(1+b_{n+1}{ }^{2} v^{2}\right)
$$

in $\boldsymbol{Z}_{p}[v] /\left(v^{n}\right)$.

Proof. By Lemma 2.2 and the hypothesis, the $\bmod p$ reduction of the total Pontrjagin class of $\mathrm{re}(\gamma)$ is equal to that of

$$
\operatorname{re}\left(\gamma^{b_{1}}\right) \oplus \operatorname{re}\left(\gamma^{b_{2}}\right) \oplus \ldots \oplus \operatorname{re}\left(\gamma^{b+1}\right) \text {, }
$$

where $\gamma$ is the canonical line bundle over $L^{2 n-1}(p)$. Let $\xi$ be the canonical line bundle over $C P(n-1)$, then the induced bundle $\pi^{*}(\xi)$ over $L^{2 n-1}(p)$ is clearly the line bundle $\gamma$, where $\pi: L^{2 n-1}(p) \longrightarrow$ $C P(n-1)$ is the natural projection. Note that $H^{*}\left(C P(n-1) ; \mathbb{Z}_{p}\right) \simeq \mathbb{Z}_{p}$ $[w] /\left(w^{n}\right)$. The Gysin sequence of the principal bundle $S^{1} \longrightarrow L^{2 n-1}(p)$ $\longrightarrow C P(n-1)$ with $Z_{p}$ coefficients is

$$
\begin{aligned}
& \longrightarrow H^{1}(C P(n-1)) \stackrel{\pi^{*}}{\longrightarrow} H^{1}\left(L^{2 n-1}(p)\right) \longrightarrow H^{0}(C P(n-1)) \\
& \longrightarrow H^{2}(C P(n-1)) \stackrel{\pi^{*}}{\longrightarrow} H^{2}\left(L^{2 n-1}(p)\right) \longrightarrow H^{1}(C P(n-1)),
\end{aligned}
$$

in which $H^{2}(C P(n-1)) \stackrel{\pi^{*}}{\longrightarrow} H^{2}\left(L^{2 n-1}(p)\right)$ must be an isomorphism. By the naturality of Chern classes,

$$
c_{1}(\gamma)=c_{1}\left(\pi^{*}(\xi)\right)=\pi^{*}\left(c_{1}(\xi)\right)=\pi^{*}(w)=v 。
$$

The first Pontrjagin class $P_{1}(\mathrm{re}(\gamma))$ comes from the identity

$$
1-P_{1}(\mathrm{re}(\gamma))=\left(1-c_{1}(\gamma)\right)\left(1+c_{1}(\gamma)\right)=1-v^{2} .
$$

Hence, the total Pontrjagin class of $\operatorname{re}(\gamma)$ in $\bmod p$ is $P(\operatorname{re}(\gamma))=1+$ $P_{1}(\operatorname{re}(\gamma))=1+v^{2}$. Since $c_{1}(\mu \otimes \nu)=c_{1}(\mu)+c_{1}(\nu)$ for any line bundles $\mu, \nu$, 


$$
P_{1}\left(\operatorname{re}\left(\gamma^{b_{j}}\right)\right)=\left(c_{1}\left(\gamma^{b_{j}}\right)\right)^{2}=\left(b_{j} c_{1}(\gamma)\right)^{2}=b_{j}^{2} \gamma^{2} .
$$

Therefore, $P\left(\operatorname{re}\left(\gamma^{b}\right)\right)=1+b_{j}^{2} v^{2}$, and

$$
\begin{aligned}
1+v^{2} & =P(\operatorname{re}(\gamma)) \\
& =P\left(\operatorname{re}\left(\gamma^{t_{1}}\right) \oplus \operatorname{re}\left(\gamma^{b_{2}}\right) \oplus \ldots \oplus \operatorname{re}\left(\gamma^{b_{n+1}}\right)\right) \\
& =P\left(\operatorname{re}\left(\gamma^{b}\right)\right) \cdot P\left(\operatorname{re}\left(\gamma^{b_{2}}\right)\right) \cdots P\left(\operatorname{re}\left(\gamma^{b+1}\right)\right) \\
& =\left(1+b_{1}^{2} v^{2}\right)\left(1+b_{2}^{2} v^{2}\right) \cdots\left(1+b_{n+1}{ }^{2} v^{2}\right)
\end{aligned}
$$

in $\mathbb{Z}_{p}[v] /\left(v^{n}\right)$, by the product formular of the Pontrjagin class.

From theorem 2.1, one can also get the total Pontrjagin and Stiefel-Whitney classes of the space $L(p ; w ; b)$.

Corollary 2.4.

$$
\begin{aligned}
& P(L(p ; w ; b))=\left(1+v^{2}\right)^{-1} \prod_{i=1}^{n+1}\left(1+b_{i}^{2} v^{2}\right), \\
& w(L(p ; w ; b))=(1+u)^{-1} \prod_{i=1}^{n+1}\left(1+b_{i} u\right),
\end{aligned}
$$

where $v$ is a prefered generator for $H^{2}(L(p ; w ; b) ; \mathbb{Z})$, so that the total Chern class of $\gamma$ is $1+v$, and $u$ is its $\bmod 2$ reduction.

In $[5], \widetilde{K O}\left(L^{2 n-1}(p)\right)$ is computed. Setting $\overline{\boldsymbol{\sigma}}=\mathrm{re}(\gamma)-2$, the $p$ torsion part of $\widetilde{K O}\left(L^{2 n-1}(p)\right)$ is a direct summand of cyclic groups generated by $\bar{\sigma}^{i}, 1 \leq i \leq(p-1) / 2$, where if $n-1=s(p-1)+r, 0 \leq r<$ $p-1$, the order of $\bar{\sigma}^{i}$ is $p^{s+1}$ for $i \leq[r / 2]$ and $p^{s}$ for $i>[r / 2]$.

Lemma 2.5. If $L(p ; w ; b)$ is stably parallelizable, then $n-1$ is less than $p$.

Proof. Let $L(p ; w ; b)$ be stably parallelizable, then $\operatorname{re}(\gamma)$ is stably isomorphic to

$$
\mathrm{re}\left(\gamma^{b_{1}}\right) \oplus \mathrm{re}\left(\gamma^{b_{2}}\right) \oplus \ldots \oplus \mathrm{re}\left(\gamma^{b+1}\right)
$$

over the standard lens space $L^{2 n-1}(p)$, which gives

$$
\operatorname{re}(\gamma)-2=\left(\operatorname{re}\left(\gamma^{b_{1}}\right)-2\right)+\ldots+\left(\operatorname{re}\left(\gamma^{b_{n+1}}\right)-2\right)
$$

in $\widetilde{K O}\left(L^{2 n-1}(p)\right)$. Since $\widetilde{K O}\left(L^{2 n-1}(p)\right)$ is abelian, we can assume that $b_{1} \leq b_{2} \leq \ldots \leq b_{n+1}$. By taking the diffeomorphic copy of $L(p ; w ; b)$ under the complex conjugation of the $i$-th coordinate, if it is needed, 
we may assume that $b_{1} \leq b_{2} \leq \ldots \leq b_{n+1} \leq(p-1) / 2$. Let $n-1=s(p-1)$ $+r, 0 \leq r<p-1$, and set $\bar{\sigma}=\mathrm{re}(\gamma)-2$, then $\bar{\sigma}^{i}, 1 \leq i \leq(p-1) / 2$, are generators of the cyclic subgroups of the $p$-torsion part of $\widetilde{K O}\left(L^{2 n-1}(p)\right)$, and their orders are $p^{s}$ or $p^{s+1}$. On the other hand,

$$
\left(\operatorname{re}\left(\gamma^{b_{1}}\right)-2\right)+\left(\operatorname{re}\left(\gamma^{b_{2}}\right)-2\right)+\ldots+\left(\operatorname{re}\left(\gamma^{b_{n+1}}\right)-2\right)
$$

can be written as a polynomial of $\bar{\sigma}$. So, we can set

$$
\bar{\sigma}=\alpha_{b_{n+1}}+\alpha_{b_{n+1}-1} \bar{\sigma}+\ldots+\alpha_{0} \bar{\sigma}^{b+1}
$$

with some coefficients $\alpha_{i}$ 's, so that $\alpha_{b_{n+1}-1}=1\left(\bmod p^{s}\right)$, and all other coefficients are divided by $p^{\text {s. }}$. And $\alpha_{0}$ is also the number of $b_{j}$ 's such that $b_{j}=b_{n+1}$ in $b_{1} \leq b_{2} \leq \ldots \leq b_{n+1}$, because

$$
\operatorname{re}\left(\gamma^{b}{ }^{n+1}\right)-2=\bar{\sigma}^{b} n+1+\text { terms of lower degree of } \bar{\sigma} \text {. }
$$

Similarly, for any $b$ with $1<b \leq(p-1) / 2$, the number of copies of $\operatorname{re}\left(\gamma^{b}\right)-2$ in

$$
\left(\operatorname{re}\left(\gamma^{b_{1}}\right)-2\right)+\left(\operatorname{re}\left(\gamma^{b_{2}}\right)-2\right)+\ldots+\left(\operatorname{re}\left(\gamma^{b_{n+1}}\right)-2\right)
$$

must be divided by $p^{s}$. Now, let $\beta$ be the number of copies of re $(\gamma)-2$ in

$$
\left(\operatorname{re}\left(\gamma^{b_{1}}\right)-2\right)+\left(\operatorname{re}\left(\gamma^{b_{2}}\right)-2\right)+\ldots+\left(\operatorname{re}\left(\gamma^{b_{n+1}}\right)-2\right),
$$

then $\beta+\beta^{\prime}=\alpha_{b_{n+1}-1}=1\left(\bmod p^{s}\right)$, where $\beta^{\prime}$ is the coefficient of $\bar{\sigma}$ in the polynomial of $\bar{\sigma}$ for

$$
\left(\operatorname{re}\left(\gamma^{b_{1}}\right)-2\right)+\ldots+\left(\operatorname{re}\left(\gamma^{b} n\right)-2\right)-\beta(\operatorname{re}(\gamma)-2) .
$$

On the other hand, $\beta^{\prime}$ is divided by $p^{s}$, so $\beta=1\left(\bmod p^{s}\right)$. Since the total number of $b_{i}$ 's is $n+1, \beta+h p^{s}=n+1$ for some $h$, so $n=s(p-1)$ $+r+1\left(\bmod p^{s}\right)$. The only possibility is $s=0$, or $s=1$ and $r=0$. In both cases, $n-1$ is less than $p$.

The next lemma will be useful to prove the main theorem.

Lemma 2. $([3])$. Let $\xi, \eta$ be oriented vector bundles over a finite $C W$ complex $X$, and suppose that

i) $\operatorname{dim}(X)<2 p+2, p$ an odd prime, and

ii) $H^{4 *}(X ; \mathbb{Z})$ has no $q$-torsion for any $q<p$.

If their Pontrjagin classes $P(\xi), P(\eta)$ are equal, then $(\xi-\eta)-(\operatorname{dim} \xi-$ $\operatorname{dim} \eta) \in \widetilde{K O}(X)$ is a 2-torsion element. 
Theorem 2.7. The space $L(p ; w ; b)$ is stably parallelizable if and only if

i) $n-1$ is less than $p$, and

ii) $\left(1+b_{1}{ }^{2} v^{2}\right)\left(1+b_{2}^{2} v^{2}\right) \cdots\left(1+b_{n+1}{ }^{2} v^{2}\right)=1+v^{2}$ in $\mathbb{Z}_{p}[v] /\left(v^{n}\right)$, or equivalently

$$
b_{1}^{2 j}+b_{2}^{2 j}+\ldots+b_{n+1}^{2 j}=1(\bmod p) \quad \text { for } j=1,2, \ldots,[(n-1) / 2]
$$

Proof. The "only if" part comes from Lemmas 2.3-2.5. Let us assume i) and ii). Then, the $\bmod p$ Pontrjagin class of re $(\gamma)$ is equal to that of $\operatorname{re}\left(\gamma^{b_{1}}\right) \oplus \operatorname{re}\left(\gamma^{b_{2}}\right) \oplus \ldots \oplus \operatorname{re}\left(\gamma^{b_{n+1}}\right)$. By Lemma 2.6,

$$
\text { re }\left(-r \oplus r^{b_{1}} \oplus r^{b_{2}} \oplus \ldots \oplus r^{b n+1}\right)-2 n
$$

is a 2 -torsion element in $\widetilde{K O}\left(L^{2 n-1}(p)\right)$. But it is clearly in the image of

$$
\text { re: } \tilde{K}\left(L^{2 n-1}(p)\right) \longrightarrow \widetilde{K O}\left(L^{2 n-1}(p)\right) \text {, }
$$

which does not contain any 2-torsion element. So it must be a zero element. Therefore, $\operatorname{re}(\gamma)$ is stably isomorphic to $\operatorname{re}\left(\gamma^{b_{1}}\right) \oplus \operatorname{re}\left(\gamma^{b_{2}}\right) \oplus \cdots$ $\oplus \operatorname{re}\left(\gamma^{b+1}\right)$ over $L^{2 n-1}(p)$, and $L(p ; w ; b)$ is stably parallelizable.

\section{§3. Some Examples}

Milnor and Orlik [9] gave the computation of $\Delta(1)$ as follows: Let $\boldsymbol{C}^{*}=\boldsymbol{C}-\{0\}$ denote the group with the multiplication. To each monic polynomial

$$
\left(t-\alpha_{1}\right)\left(t-\alpha_{2}\right) \cdots\left(t-\alpha_{k}\right), \alpha_{i} \in C^{*},
$$

assign the divisor

$$
\begin{aligned}
\text { divisor } & \left(\left(t-\alpha_{1}\right)\left(t-\alpha_{2}\right) \cdots\left(t-\alpha_{k}\right)\right) \\
= & \left.\left\langle\alpha_{1}\right\rangle+\left\langle\alpha_{2}\right\rangle+\cdots+<\alpha_{k}\right\rangle
\end{aligned}
$$

as an element of the rational group ring $\boldsymbol{Q} \boldsymbol{C}^{*}$. Denote

$$
\begin{aligned}
\Lambda_{k} & =\operatorname{divisor}\left(t^{k}-1\right) \\
& =\langle 1\rangle+\langle\xi\rangle+\ldots+\left\langle\xi^{k-1}\right\rangle,
\end{aligned}
$$

where $\xi=e^{2 \pi i / k}$. Note that $\Lambda_{a} \Lambda_{b}=(a, b) \Lambda_{[a, b]}$, where $[a, b]$ denotes their least common multiple and $(a, b)$ the greatest common divisor. Then, for a weighted homogeneous polynomial $f\left(z_{1}, z_{2}, \ldots, z_{n+1}\right)$ of type $w=\left(w_{1}, w_{2}, \ldots, w_{n+1}\right)$, the characteristic polynomial $\Delta(t)=$ determinant 
$\left(t I_{*}-h_{*}\right)$ of the linear transformation $h_{*}: H_{n}(F ; \boldsymbol{C}) \longrightarrow H_{n}(F ; \boldsymbol{C})$ is determined by

$$
\text { divisor } \Delta=\left(v_{1}^{-1} \Lambda_{u_{1}}-1\right)\left(v_{2}^{-1} \Lambda_{u_{2}}-1\right) \ldots\left(v_{n+1}{ }^{-1} \Lambda_{u_{n+1}}-1\right) \text {, }
$$

where $w_{i}=u_{i} / v_{i}, i=1,2, \ldots, n+1$, is the expression in irreducible form.

To make the computation of $\Delta(1)$ easy, we cite two Milnor-Orlik's theorems.

Theorem 3.1 ([9]). By using $\Lambda_{a} \Lambda_{b}=(a, b) \Lambda_{[a, b]}$, divisor $\triangle$ can be expressed as a linear combination of the divisors $\Lambda_{r}$. Let

$$
\text { divisor } \Delta=a_{1} \Lambda_{1}+a_{2} \Lambda_{2}+\ldots+a_{s} \Lambda_{s} \text {, }
$$

and define two numbers $k(\Delta)$ and $\rho(\Delta)$ by the formular

$$
k(\Delta)=a_{1}+a_{2}+\ldots+a_{s} \text {, and } \rho(\Delta)=2^{a_{2} 3^{a_{3}}} \ldots s^{a_{s}} .
$$

Then, $k(\Delta)$ and $\rho(\Delta)$ are non-negative integers, and

$$
\begin{array}{ll}
\Delta(1)=\rho(\Delta) & \text { if } k(\Delta)=0, \\
\Delta(1)=0 & \text { if } k(\Delta) \neq 0 .
\end{array}
$$

Theorem 3.2 ([9]). Let

$$
f\left(z_{1}, \ldots, z_{n+1}\right)=f_{1}\left(z_{1}, \ldots, z_{k}\right)+f_{2}\left(z_{k+1}, \ldots, z_{n+1}\right)
$$

where $f_{1}$ and $f_{2}$ are weighted homogeneous polynomials, and let $\Delta_{1}$ and $\Delta_{2}$ be the characteristic polynomials associated to $f_{1}$ and $f_{2}$. For the weight $w=$ $\left(w_{1}, \ldots, w_{k}, \ldots, w_{n+1}\right)$, express $w_{i}=u_{i} / v_{i}, i=1,2, \ldots, n+1$, in an irreducible form. Suppose that each of the numbers $u_{1}, \ldots, u_{k}$ is relatively prime to each of $u_{k+1}, \ldots, u_{n+1}$. Then the numbers $k(\Delta), \rho(\Delta)$ corresponded to the polynomial $f=f_{1}+f_{2}$ are determined by the integers $k_{j}=k\left(\Delta_{j}\right)$ and $\rho_{j}=$ $\rho\left(\Delta_{j}\right)$ corresponded to $f_{j}, j=1,2$ according to the formulars

$$
k(\Delta)=k_{1} k_{2} \text { and } \rho(\Delta)=\rho_{1}^{k_{2}} \rho_{2}^{k_{1}} .
$$

The next theorems show how one can construct topological spheres using the weighted homogeneous polynomial.

Theorem 3.3. Let $g\left(z_{1}, z_{2}, \ldots, z_{m}\right)$ be a weighted homogeneous polynomial with weight $w=\left(w_{1}, w_{2}, \ldots, w_{m}\right), w_{i}=u_{i} / v_{i}$ as before, $i=1,2, \ldots$, $m$. Choose any two positive integers $w_{m+1}$ and $w_{m+2}$ such that $\left(w_{m+j}, u_{i}\right)=1$ for all $i=1,2, \ldots, m ; j=1,2$. Then a polynomial $f$ defined by 


$$
f\left(z_{1}, \ldots, z_{m}, z_{m+1}, z_{m+2}\right)=g\left(z_{1}, \ldots, z_{m}\right)+z_{m+1}{ }^{w_{m+1}}+z_{m+2}{ }^{w_{m+2}}
$$

is also a weighted homogeneous polynomial of weight $\left(w_{i}\right)$, and $\sum_{w}=$ $f^{-1}(0) \cap S^{2 m+3}$ is a topological sphere.

Proof. Let $k, k(g), k_{1}, k_{2}$ and $\rho, \rho(g), \rho_{1}, \rho_{2}$ be numbers defined in Theorem 3.1 associated to $f, g, z_{m+1}{ }^{w_{m+1}}, z_{m+2}{ }^{w_{m+2}}$ respectively. Clearly, divisor $\Delta_{i}=A_{w_{i}}-1$, for $i=1,2$, so that $k_{i}=1-1=0$. Hence $k=k_{1} k_{2} k$ $(g)=0$, and then $\Delta(1)=\rho=\left(\rho_{g}{ }^{k_{1}} \rho_{1}{ }^{k(g)}\right)^{k_{2}} \rho_{1}{ }^{k(g) k_{1}}=1$. Therefore, $\sum_{w}$ is a topological sphere.

Theorem 3.4 ([11])。Let $g(z)$ be a weighted homogeneous polynomial in $\mathbb{C}^{n}$ with an isolated critial point at the origin, and let $f(z, w)$ be a weighted homogeneous polynomial in $\mathbb{C}^{n} \times \mathbb{C}^{2}$ defined by $f(z, w)=g(z)+w_{1} w_{2}$. Then $g^{-1}(0) \cap S^{2 n-1}$ is a topological sphere if and only if $f^{-1}(0) \cap S^{2 n+3}$ is a topological sphere. (Here, $n>3$ ).

We conclude with an example. Let

$$
f\left(z_{1}, z_{2}, \ldots, z_{7}\right)=f_{1}\left(z_{1}, \ldots, z_{5}\right)+f_{2}\left(z_{6}, z_{7}\right),
$$

where

$$
\begin{aligned}
& f_{1}\left(z_{1}, z_{2}, \ldots, z_{5}\right)=z_{1}{ }^{3}+z_{2}{ }^{6 k-1}+z_{3}{ }^{2}+z_{4}{ }^{2}+z_{5}{ }^{2} \\
& f_{2}\left(z_{6}, z_{7}\right)=z_{6} z_{7} .
\end{aligned}
$$

Then, $f$ is a weighted homogeneous polynomial with weight $\left(w_{i}\right)=$ $(3,6 k-1,2,2,2,1 / 2,1 / 2)$. By Theorem 3.4, $\sum_{w}=f^{-1}(0) \cap S^{13}$ is an 11-dimensional topological sphere. First, we are interested in the diffeomorphic type of this sphere $\sum_{w}$. Let $F, F_{1}$, and $F_{2}$ be the fibre in the Milnor's fibering corresponding to the polynomials $f_{9} f_{1}$, and $f_{2}$ respectively. Then $F, F_{1}$, and $F_{2}$ are diffeomorphic to $f^{-1}(1), f_{1}^{-1}(1)$, and $f_{2}^{-1}(1)$ respectively (cf. [8], Lemma 9.4.), and $f^{-1}(1)$ is homotopy equivalent to the join $f_{1}^{-1}(1) * f_{2}^{-1}(1)$ (cf. [11]). Note that $f_{2}^{-1}(1)$ has the same homotopy type as $S^{1}$. Hence,

$$
\begin{aligned}
H_{6}(F ; \mathbb{Z}) & =H_{6}\left(F_{1} * F_{2} ; \mathbb{Z}\right) \\
& =\sum_{i+j=5} \tilde{H}_{i}\left(F_{1} ; \mathbb{Z}\right) \otimes \tilde{H}_{j}\left(F_{2} ; \mathbb{Z}\right) \bigoplus_{p+q=4} \tilde{H}_{p}\left(F_{1} ; \mathbb{Z}\right) * \tilde{H}_{p}\left(F_{2} ; \mathbb{Z}\right) \\
& =H_{4}\left(F_{1} ; \mathbb{Z}\right) \otimes H_{1}\left(F_{2} ; \mathbb{Z}\right)=H_{4}\left(F_{1} ; \mathbb{Z}\right) .
\end{aligned}
$$

(See [7] for the 2nd isomorphism). Hence, the signature of the intersection pairing of $F$ is equal to that of $F_{1}$. Also it is well-known 
that $f_{1}^{-1}(0) \cap S^{9}=k \cdot g_{2}$ and the signature of $F_{1}$ is equal to $8 k$, where $g_{2}$ is a generator of the cyclic group of all 28 7-dimensional homotopy spheres. Therefore, we get $\sum_{w}=\int^{-1}(0) \cap S^{13}=k \cdot g_{3}$ for a generator of the cyclic group of all 992 11-dimensional homotopy spheres.

To get a cyclic group action on these spheres which induces stably parallelizable orbit spaces, it is required to choose a prime $p$ and numbers $b_{1}$ 's such that

$$
\begin{array}{ll}
w_{1} b_{1}=w_{2} b_{2}=\ldots=w_{7} b_{7} & (\bmod p), \\
b_{1}{ }^{2}+b_{2}{ }^{2}+\ldots+b_{7}{ }^{2}=1 & (\bmod p), \\
b_{1}{ }^{4}+b_{2}{ }^{4}+\ldots+b_{7}{ }^{4}=1 & (\bmod p) .
\end{array}
$$

Hence,

$$
\begin{array}{ll}
(1 / 3)^{2}+b_{2}{ }^{2}+3(1 / 2)^{2}=-7 & (\bmod p), \\
(1 / 3)^{4}+b_{2}{ }^{4}+3(1 / 2)^{4}=-31 & (\bmod p)
\end{array}
$$

must be satisfied. Accordingly, $120524=0(\bmod p)$, so $p=29$ or $p=$ 1039.

For example, if $p=29$, then we can take

$$
\left(b_{1}, b_{2}, \ldots, b_{7}\right)=(10,1,15,15,15,2,2),
$$

and then, for $k=10+29 q, q=1,2, \ldots, 992, \sum_{w}$ represent all $99211-$ dimensional homotopy spheres. Furthermore, on these 992 homotopy spheres, the cyclic group action defined by the given $b_{\imath}$ 's is well defined, and all their orbit spaces are stably parallelizable.

\section{Bibliography}

[1] Atiyah, M. F., K-theory, Benjamin Inc., New York, 1967.

[2] Brieskorn, E., Beiespiele zur Differential-Topologie von Singulatitaten, Inventiones Math., 2 (1966), 1-14.

[ 3 ] Ewing, J., S. Moolgavkar, L. Smith and R.S. Stong, Stable parallelizability of lens spaces, $J$. of Pure and Applied Algebra, 10 (1977), 177-191.

[4] Hirzebruch, F., Singularities and exotic spheres, Séminaire Bourbaki, 19e année, (1966/67), no 314.

[5] Kambe, T., The structure of $K_{\Lambda}$-rings of the lens space and their applications, $J$. Math. Soc. Japan, 18 (1966), 135-146.

[6] Kervaire M. and J. Milnor, Groups of homotopy spheres I, Annals of Math., 77 (1963), 504-537.

[7] Milnor J., Constructions of universal bundle II, Annals of Math., 63 (1956), 430-436.

[8] Singular points of complex hypersurfaces, Annals of Math., Studies 61, Princeton University Press, New Jersey, 1968.

[9] and P. Orlik, Isolated singularities defined by weighted homogeneous polynomials, Topology, 9 (1969), 385-393. 
[10] and J. Stasheff, Characteristic Classes, Annals of Math., Studies 76, Princeton Univ. Press, New Jersey, 1974.

[11] Oka M., On the homotopy types of hypersurfaces defined by weighted homogeneous polynomials, Topology, 12 (1973), 19-32.

[12] Orlik P., Smooth homotopy lens spaces, Michigan Math. J., 16 (1969), 245-255. 